\title{
Hair Coat Color
}

National Cancer Institute

\section{Source}

National Cancer Institute. Hair Coat Color. NCI Thesaurus. Code C90392.

The hue of a subject's hair or fur. 